\title{
Práticas Criativas em Sala de Aula e a Criatividade dos Docentes: Estudo Exploratório no Ensino Básico
}

\author{
Creative Practices in the Classroom and the Creativity of \\ Teachers: An Exploratory Study in Elementary Schools
}

\author{
Maria de Fátima Morais ${ }^{1 *}$ \\ Lúcia C. Miranda ${ }^{2}$ \\ ${ }^{1}$ Universidade do Minho, Portugal \\ ${ }^{2}$ Universidade da Madeira, Portugal
}

\begin{abstract}
Criatividade tem sido requerida para as práticas dos professores em sala de aula, mas esta associação necessita de maior avaliação. Também os conceitos de pessoa e de docente criativos são distintos, mas tal distinção é mais explorada teórica do que empiricamente. Este estudo, ainda exploratório, analisa a relação entre autoavaliação de criatividade feita por docentes e práticas criativas em sala de aula por eles percecionadas. Para avaliar as práticas, usou-se o Inventário de Práticas Docentes para a Criatividade em Educação Superior (Morais et al., 2014). Para avaliar a criatividade dos docentes usou-se a Escala de Personalidade Criativa (Garcês et al., 2015). Participaram 111 docentes dos $2^{\circ}$ e $3^{\circ}$ ciclos do Ensino Básico de Portugal. Maioritariamente eram mulheres, tinham entre 31 e 61 anos e com experiência docente entre 4 e 37 anos. Os resultados mostraram correlações estatisticamente significativas entre dimensões do Inventário de Práticas Docentes Criativas e a Escala unifatorial da Personalidade Criativa. Uma análise da variância multivariada demonstrou diferenças estatisticamente significativas nas práticas docentes em função da criatividade dos docentes, havendo oscilações nestas práticas em grupos discrepantes (docentes mais e menos criativos). Os dados poderão ser úteis para a investigação e formação de professores, nomeadamente para reflexões e práticas.
\end{abstract}

Palavras-chave: Criatividade; Práticas em sala de aula; Avaliação de professores; Personalidade.

Creativity has been required for teacher practices in the classroom, but this association needs further evaluation. Also, the concepts of creative person and creative teacher are distinguished, but this distinction is more explored theoretical than empirically. This exploratory study analyzes the relationship between selfassessment of creativity made by teachers and creative practices in the classroom perceived by them. To evaluate the practices, the Inventory of Teaching Practices for Creativity in Higher Education (Morais et al., 2014) was used. To assess teachers' creativity, the instrument was Creative Personality Scale (Garcês et al., 2015). The sample was 111 teachers from the 2nd and 3rd cycles of Basic Education in Portugal. Mostly they were women, with ages between 31 and 61 years old and exercised the teaching function between 4 and 37 years. The results showed statistically significant correlations between dimensions of the Inventory of Creative Teaching Practices and the unifactorial Creative Personality Scale. The multivariate analysis of variance showed statistically significant differences in teaching practices due to the creativity of teachers, with fluctuations in creative practices in different groups (more and less creative teachers). The data may be useful for the investigation and the training of teachers, namely for reflections and practices.

Keywords: Creativity; Teacher evaluation; Classroom practices; Personality.

*Contacto: famorais@ie.uminho.pt

ISSN: $1696-4713$

www.rinace.net/reice/

revistas.uam.es/reice
Recibido: $\quad 30$ de abril 2020

$1^{\text {a }}$ Evaluación: 13 de julio 2020

$2^{\text {a }}$ Evaluación: 27 de septiembre 2020

Aceptado: 16 de noviembre 2020 


\section{Revisão da Literatura}

Entende-se criatividade como a conjugação da raridade e da eficácia, quer seja na resolução de um problema ou na expressão de uma mensagem num dado momento sociocultural (Kaufman, 2016; Runco e Jager, 2012). Em qualquer contexto profissional ou pessoal é estudada e afirmada a relevância das competências criativas (Ahmadi e Besançon, 2017; Williams, Runco e Berlow, 2016), sendo mesmo consideradas um requisito da inovação (Lubart e Zesnani, 2010). Ora, atualmente não há progresso sem inovação (Lambert, 2017).

O contexto educativo, especificamente o escolar, surge então como fundamental para a preocupação com o desenvolvimento de competências criativas nos alunos (Alencar, 2015; Soulé e Warrick, 2015), considerando-se a criatividade um potencial disponível em todos os indivíduos (Runco, 2014) e estando em causa, neste caso, a criatividade media (midlleC) a partir do modelo de Kaufman e Beghetto (2008). A promoção da criatividade na Educação, paralelamente ou de forma infundida face ao currículo, deve ser uma premissa atual, considerando todos os níveis de aprendizagem no percurso escolar (Craft e Jeffrey, 2010; Cropley, 2015). Esta premissa deve ainda ser transversal aos vários domínios do conhecimento, estando a emergir pesquisas e intervenções em áreas como a Tecnologia, as Artes, a Educação, a Filosofia, as Letras ou a Matemática (Baer, 2017; Cropley e Cropley, 2009; Higuchi et al., 2016; Seckel et al., 2019). O trabalho de pesquisa, no campo da criatividade na escola, tem contemplado o estudo de perceções quer de docentes quer de alunos (Morais e Almeida, 2019; Raso e Arando, 2019; Seckel et al., 2019), tal como o estímulo ao desempenho criativo, mais uma vez tomando diferentes faixas etárias (Azevedo, Morais e Martins, 2017; Catarino et al., 2019; Monteiro, 2020). A escola e os docentes têm de estar preparados para os desafios que os alunos encontram e encontrarão no séc. XXI (European Commission, 2015; Ministério da Educação, 2017; Patston et al., 2017). Neste contexto, o professor surge compreensivelmente com um papel essencial enquanto modelo de atitudes e de comportamentos, nomeadamente a nível criativo (Cremin, 2009; Glaveanu, 2018; Miranda e Morais, 2019; Omdal e Graefe, 2017).

Parece, então, pertinente refletir sobre dois conceitos que, distintos, partilharão fronteiras importantes: professor com características pessoais criativas e professor que intencionalmente promove nos seus alunos a criatividade. Este último foi distinguido como sendo o professor criativo por Craft (Craft, 2005; Craft e Jefrey, 2008) e aquele que deve ser alvo de atenção para a escola se tornar num espaço promotor de criatividade (Cropley, 2015). Quando se aborda este conceito, parecem emergir duas perspetivas, uma que aponta as práticas (as tarefas, a gestão dos conteúdos, as dinâmicas relacionais) indicadoras de um clima criativo, ou seja, facilitador de criatividade (Cremin, 2009; Miller e Dumford, 2016) que, neste caso, o docente deve gerir intencionalmente em sala de aula. A outra perspetiva refere características que o docente deve possuir pessoalmente para que a gestão anterior seja possível ou facilitada e que correspondem a características de uma personalidade criativa (Chávez-Eakle, Eakle e Cruz-Fontes, 2012; Feist, 2017; Miranda e Morais, 2019; Morais, 2013). Cada uma destas perspetivas irá ser brevemente analisada, já que o que interessa para este trabalho é a relação entre ambas.

O que é um clima criativo em sala de aula? Que práticas devem os docentes tomar em consideração para que o processo de ensino-aprendizagem e o espaço de sala de aula sejam fomentadores de competências criativas nos alunos? A diversidade de abordagens, a gestão dos conteúdos e das tarefas tendo em conta os interesses e vivências dos alunos, o 
entusiasmo pela docência, a competência científica demonstrada pelo docente, a sua disponibilidade interpessoal face aos alunos na sala de aula e mesmo fora dela, são exemplos de características que devem operacionalizar um ensino que tem a criatividade dos alunos como objetivo intencional e paralelo ao de conteúdos escolares (Ahmadi e Besançon, 2017; Beghetto e Kaufman, 2014; Cropley, 2015; Starko, 2014).

Também a estimulação da curiosidade, da autonomia, da autoconfiança do aluno, por parte do docente, assim como valorizar e incentivar a expressão de ideias, a divergência, as flexibilidades em pontos de vista corporizam esse clima propiciador de criatividade. As atitudes e realizações criativas devem ainda ser valorizadas explicitamente (Alencar e Sobrinho, 2015, Fleith e Morais, 2017).

Como condições facilitadoras de um clima criativo pode-se ainda referir a segurança psicológica criada em sala de aula, por sua vez permissora de riscos e de erros. E tal perceção de segurança ajuda ainda à autonomia, à crítica e à participação em geral. Os conteúdos abordados, assim como as tarefas e estratégias do trabalho em sala de aula devem evitar a rotina, reforçando-se a diversidade e a articulação com interesses dos alunos em causa. Estas condições criadas no quotidiano escolar suscitarão então o compromisso, a liberdade de criar e a motivação dos seus participantes (Cropley, 2015; Kampylis e Berki, 2014; Sierra et al., 2015).

Por seu lado, quem cria, gere e avalia o clima criado em sala de aula é o docente. Logo, as características pessoais deste docente estarão em causa para tais condições facilitadoras de criatividade serem por ele operacionalizáveis na prática pedagógica, embora de forma independente das suas próprias realizações criativas (Fryer, 2005; Miranda e Morais, 2019). Como é que as características pessoais do docente, incluindo nelas uma personalidade mais ou menos criativa, se projetam nas práticas docentes mais e menos criativas que ele pode desenvolver com os seus alunos? Como é que um docente que estimula a autonomia ou o risco nos alunos, cria alternativas em sala de aula, mantém um clima de compromisso e de prazer, poderia não ter ele próprio, por exemplo, autoconfiança, tolerância à ambiguidade ou uma elevada motivação no que faz?

Desde a década de 60 foram identificadas características de uma personalidade típica das pessoas criativas (Barron, 1969; MacKinnon, 1978). Ter uma personalidade criativa obviamente não é garantia de ser criativo, mas o contrário afirmou-se verdadeiro nestes estudos empíricos. Ser criativo implica, assim, ter características como, por exemplo, autonomia, autoconfiança, abertura à experiência, tolerância à ambiguidade, facilidade em arriscar, paixão pelo que se faz ou persistência (Barbot, Besançon e Lubart, 2015; Costa et al., 2015). Estas características não são independentes. Por exemplo, a autonomia é facilitadora de elevada energia e motivação intrínseca (Grohaman e Snyder, 2017), enquanto a paixão pelo que se faz se associa à persistência, ao compromisso em profundidade e em tempo nas tarefas (Miranda e Morais, 2019; Moeller, Keiner e Grassinger, 2015). Ryan e Deci (2017) aliam, por exemplo ainda, a autonomia à motivação intrínseca, já que quanto maior controlo se sente sobre uma tarefa, maior prazer se coloca na sua realização.

Foca-se agora um pouco mais algumas destas características criativas, tal como foram exemplificadas anteriormente práticas criativas necessárias em sala de aula. Growham e Snyder (2017), indo ao encontro dos trabalhos clássicos de Amabile (1983, 1996), afirmam que é uma elevada motivação, a paixão pela tarefa a realizar, que distingue o que se pode fazer e o que se faz de facto numa tarefa criativa. São então características pertencentes a 
uma personalidade criativa (Andreas et al., 2016; Csíkszentmihályi, 2014; Kaufman e Gregoire, 2015).

Enquadram-se aqui os estudos, clássicos e recentes, que afirmam uma motivação intrínseca como facilitadora da criatividade (Amabile, 1983, 1996; Ceci e Kumar, 2016; Miranda e Morais, 2019; Muñoz-Pascual e Galende, 2017), apesar de este tema não ser ainda pacífico por haver também contribuições possíveis de motivação extrínseca. Por outro lado, a abertura à experiência continua a ser uma das características de personalidade mais associadas à criatividade, permitindo a novidade, a mudança e a diversidade, pois possibilita a experimentação, o relacionamento de mais ideias e de ideias remotas, assim como a flexibilidade de não resistir em ponderar perspetivas diferentes e mesmo contraditórias (Batey e Hughes, 2017). Também não há criatividade sem autonomia ou autoconfiança, pois estas permitem a ousadia para ser original, assim como resiliência face a incompreensões de outrem perante o que se cria e é inovador (Cropley, 2015). Por sua vez, a tolerância à ambiguidade permite resistir à pressão para fechar problemas precocemente ou aparentemente sem solução; pode-se manter tais problemas em aberto mais tempo, podendo assim as soluções serem potencialmente mais criativas (Robinson, Workman e Freeburg, 2018). A persistência, associada à tolerância à ambiguidade, surge ainda como essencial na resolução de problemas criativos, mantendo o processo, mesmo que este seja complicado ou prolongado (Starko, 2014). No caso do docente, tais características, apesar de não definirem um professor criativo (Craft, 2005), são compreensivelmente necessárias para a garantia do clima criativo num processo de ensino-aprendizagem.

Tem havido alguns estudos de instrumentos de avaliação de práticas criativas em sala de aula por parte de diferentes países (Davidovitch e Milgram, 2006; Fleith e Alencar, 2005; Sadeghi e Ofoghi, 2011) e o uso de Escalas para avaliar a personalidade criativa tem sido assumido até à atualidade como uma medida de criatividade (Gough, 1979; Jauk et al., 2019). Porém, a investigação sobre as práticas criativas em sala de aula sobre características de personalidade criativa necessita ainda de maior investimento, sobretudo empírico (Morais et al., 2019; Slate et al., 2011; Susanto et al., 2018), nomeadamente sobre a sua relação com os contornos de uma personalidade criativa dos docentes. É essa a preocupação a analisar neste artigo, ou seja, se existe uma associação entre características criativas de professores e as suas práticas docentes criativas, e se tais práticas variam em função da existência de maior ou menor autoperceção de características criativas por parte desses professores.

\section{Método}

A metodologia utilizada apresenta características de um estudo descritivo e exploratório e considera um paradigma quantitativo. Pretende-se explorar, descrever, analisar e compreender as associações entre características criativas de professores e as suas práticas docentes criativas, e se tais práticas variam em função da existência de maior ou menor autoperceção de características criativas por parte desses professores, conforme referido. Trata-se, pois, de um estudo de grupo ou caso único (Almeida e Freire, 2017).

\section{Participantes}

A amostra foi de conveniência, tendo participado no estudo 111 professores de duas escolas públicas do Norte de Portugal. Foram escolhidos estes docentes porque 
participavam numa investigação-ação que decorria nestas duas escolas. Todos os professores lecionavam no $3^{\circ}$ ciclo do Ensino Básico e ou no Ensino Secundário e maioritariamente eram professoras (94,2\%). Na sua maioria (79\%) apresentava idades compreendidas entre os 31 e os 61 anos. O tempo de serviço como docentes variou entre 4 ( 1 professor) e 37 anos ( 1 professor), com média de 20 anos e desvio padrão de 8,1 (36\% apresentou tempo de serviço entre 4 e 15 anos; 42,3\% entre 16 e 26 anos e $21,6 \%$ entre 27 e 37 anos de serviço).

\section{Instrumentos}

As práticas docentes foram avaliadas pelo Inventário de Práticas Docentes para a Criatividade em Educação Superior (Morais et al., 2014), após uma revisão independente dos itens por dois professores do $2^{\circ}$ e $3^{\circ}$ ciclos de escolaridade no sentido da adequação a esse contexto de escolaridade. Todos os itens foram achados pertinentes e claros, havendo poucas alterações efetuadas nos mesmos face à versão inicial. Estas alterações pontualmente disseram respeito a retirar a palavra "ampla" no item "Proporciona ampla bibliografia sobre os conteúdos abordados" e retirar a expressão “dentro e fora da sala de aula” no item "Tem disponibilidade para tirar dúvidas com os alunos dentro e fora da sala de aula”, achando assim os avaliadores ficarem itens mais adequados à população discente não universitária. O Inventário foi originalmente construído no Brasil por Alencar e Fleith (2010) e avalia a perceção que os docentes têm das suas práticas no sentido da promoção da criatividade nos alunos. É constituído por 22 itens que se organizam em quatro fatores. O fator Incentivo a Novas Ideias (alpha de Cronbach de 0,93) integra 8 itens como, por exemplo, "Desperta nos alunos o gosto pela descoberta e pela procura de novos conhecimentos", "Estimula a iniciativa dos alunos" ou "Faz perguntas desafiadoras que motivam os alunos a pensar". O fator Clima para a Expressão de Novas Ideias (alpha de Cronbach de 0,76) apresenta 5 itens como, por exemplo, "Valoriza as ideias originais dos alunos", "Cria ambiente de respeito e de aceitação pelas ideias dos alunos" ou "Escuta com atenção as intervenções dos alunos”. O fator Interesse pela Aprendizagem do Aluno, (alpha de Cronbach de 0,75), tem 5 itens como, por exemplo, "Utiliza exemplos para ilustrar o que ensina na aula”, “Tem disponibilidade para tirar dúvidas dos alunos” ou “Está atento aos interesses do aluno”. Por fim, o fator Avaliação e Metodologias de Ensino (alpha de Cronbach de 0,53) reúne apenas 4 itens como, por exemplo "Preocupa-se apenas como conteúdo informativo", "Na avaliação exige apenas a reprodução do conteúdo da aula ou do material de apoio" ou "Utiliza a mesma metodologia de ensino". As respostas estão organizadas numa escala de Likert com cinco opções (entre 1: "discordo totalmente”, a 5: “concordo totalmente”). Exceto no fator Avaliação e Metodologia de Ensino (de cotação invertida), quanto mais próximo de cinco é o valor obtido, melhor é a perceção que o docente tem das suas práticas favorecedoras de criatividade no contexto escolar.

As características criativas foram avaliadas pela Escala de Personalidade Criativa-EPC (Garcês et al., 2015), a qual é constituída por 30 itens, avaliados numa escala de Likert de 5 pontos (entre 1: “discordo totalmente”, a 5: “concordo totalmente”). Trata-se de uma escala unidimensional com um alpha de Cronbach de 0, 92. Quanto mais próximo de 150 é o valor obtido na Escala, maior é a partilha de características de uma personalidade criativa que o respondente avalia ter. Como exemplos de itens, pode-se mencionar "sou uma pessoa curiosa", "encontro motivação em tudo o que faço" ou "sou uma pessoa aberta a novas ideias". 


\section{Procedimentos na recolha e no tratamento dos dados}

Previamente ao preenchimento dos questionários todos os docentes foram informados acerca dos objetivos da investigação-ação. Foram garantidos também o anonimato e o voluntariado neste estudo. O preenchimento das Escalas foi individual. As análises estatísticas foram realizadas com recurso ao programa IBM SPSS (versão 26.0 para Windows). Antes da realização das análises, os itens relativos à subescala Avaliação e Metodologias de Ensino foram recodificados, uma vez que se apresentavam na sua forma negativa.

\section{Resultados}

O primeiro objetivo do estudo é verificar se existe uma associação entre a personalidade criativa e as práticas criativas dos docentes. Na quadro 1, são apresentados os resultados das análises estatísticas descritivas, mais concretamente a média e o desvio-padrão das variáveis em análise. São também integrados nesta quadro os valores das correlações (produto x momento de Pearson) entre as práticas criativas e a personalidade criativa dos professores.

A partir dos resultados do quadro 1, é possível referir que os docentes obtêm valores médios superiores nas dimensões relacionadas com o Incentivo a Novas Ideias e com o Clima para a Expressão de Ideias. Pode-se exemplificar com as médias mais elevadas obtidas nalguns itens pertencentes a cada uma das dimensões da Escala de Práticas Criativas (Morais et al., 2014): na dimensão Incentivo a Novas Ideias, no item $\mathrm{n}^{\mathrm{o}} 4$ "Estimula a Iniciativa dos Alunos" - obteve-se a média de 4,75 e desvio padrão $=0,46$, no item $n^{\circ} 8$ "Leva o aluno a perceber e a conhecer diferentes opiniões sobre o problema ou o tema em estudo" a média foi de 4,6 e o desvio padrão de $0,0,55$. Relativamente à dimensão Clima para a Expressão de Ideias, no item $n^{\circ} 1$ "Escuta com atenção as intervenções dos alunos" obteve-se a média de 4,8 e o desvio padrão de 0,40 e no item $n^{\circ} 11$ "Cria um ambiente de respeito e de aceitação pelas ideias dos alunos" a média foi de 4,7 e o desvio padrão de 0,48 .

Quadro 1. Resultados descritivos e correlacionais entre as dimensões da personalidade criativa e das práticas criativas

\begin{tabular}{lccc}
\hline & MédiA & $\begin{array}{c}\text { DESVIO } \\
\text { PADRÃo }\end{array}$ & $\begin{array}{c}\text { CoRRELAÇÃo DE } \\
\text { PEARSON }\end{array}$ \\
\hline Personalidade criativa & 125,1 & 11,82 & - \\
Clima Expressão Ideias & 23,0 & 1,85 & $0,43^{* *}$ \\
Interesse Aprendizagem & 18,4 & 2,27 & $0,32^{* *}$ \\
Incentivo Novas Ideias & 31,6 & 2,91 & $0,49 * *$ \\
Avaliação e Metodologias de Ensino & 9,9 & 3,63 & $-0,13$ \\
\hline
\end{tabular}

Nota: ${ }^{* *} \mathrm{p}<0,05 ;{ }^{*} \mathrm{p}<0,01$.

Fonte: Elaborado pelos autores.

Relativamente aos valores das correlações entre a personalidade criativa e as diferentes dimensões das práticas criativas, pode-se referir que as correlações assumem significado estatístico e são moderadas, com exceção da correlação entre a personalidade criativa e a dimensão Avaliação e Metodologias de Ensino da Escala de Práticas Criativas, a qual é negativa e não assume significado estatístico $(r=-0,13)$. 
Com o objetivo de investigar se as práticas criativas dos docentes variavam em função das suas características mais e menos criativas procedeu-se, previamente, à divisão dos docentes em 4 grupos (Percentis 25, 50, 75 e 90), tomando a variável personalidade criativa. No quadro 2 , apresentam-se os resultados descritivos (média e desvio padrão) das dimensões da Escala de Práticas Criativas, considerando a divisão dos docentes pelos respetivos percentis

Quadro 2. Resultados descritivos das práticas criativas de acordo com o quartil a que pertencem os docentes (personalidade criativa)

\begin{tabular}{cccccc}
\hline & $\begin{array}{c}\text { CLIMA } \\
\text { EXPRESSÃo } \\
\text { IDEIAS }\end{array}$ & $\begin{array}{c}\text { INTERESSE } \\
\text { APREND. }\end{array}$ & $\begin{array}{c}\text { INCENTIVO } \\
\text { NovAS IDEIAS }\end{array}$ & $\begin{array}{c}\text { AVALIAÇÃo E } \\
\text { MET. DE } \\
\text { ENSINO }\end{array}$ \\
\hline $\begin{array}{c}\text { Percentil 25 } \\
(\mathrm{N}=22)\end{array}$ & $\mathrm{M}(\mathrm{DP})$ & $21,8(2,30)$ & $17,5(2,72)$ & $29,7(2,87)$ & $11,5(3,57)$ \\
\hline $\begin{array}{c}\text { Percentil } 50 \\
(\mathrm{~N}=30)\end{array}$ & $\mathrm{M}(\mathrm{DP})$ & $22,7(1,66)$ & $17,9(1,86)$ & $30,9(3,04)$ & $9,0(3,01)$ \\
\hline $\begin{array}{c}\text { Percentil } 75 \\
(\mathrm{~N}=30)\end{array}$ & $\mathrm{M}(\mathrm{DP})$ & $23,1(1,61)$ & $18,7(2,25)$ & $31,7(2,66)$ & $9,6(3,29)$ \\
\hline $\begin{array}{c}\text { Percentil } 90 \\
(\mathrm{~N}=29)\end{array}$ & $\mathrm{M}(\mathrm{DP})$ & $24,2(1,14)$ & $19,3(2,03)$ & $33,7(1,57)$ & $9,7(4,33)$ \\
\hline
\end{tabular}

Fonte: Elaborado pelos autores.

Como se pode observar (cf. quiadro 2), tendencialmente, a perceção das práticas criativas aumenta à medida que aumenta a perceção de características criativas. A única exceção verifica-se na dimensão Avaliação e Metodologias de Ensino, na qual os professores mais criativos (percentil 90) apresentam valores médios inferiores aos dos seus colegas menos criativos, do percentil 25, mas superiores aos restantes percentis (percentis 50 e 75). Importa referir ainda que são os docentes do percentil 50 os que apresentam médias mais baixas nesta dimensão. Também é nesta dimensão que os desvios padrão são mais elevados.

Quadro 3. Resultados da análise da variância $F$ - Manova-one way

\begin{tabular}{|c|c|c|c|c|}
\hline & $\begin{array}{l}\text { TeSTE } \\
\mathbf{F}\end{array}$ & $\begin{array}{c}\text { SIGNIFICÂNCIA } \\
\text { ESTATÍSTICA }\end{array}$ & $\begin{array}{c}\text { TAMANHO DO } \\
\text { EFEITO N }^{2} \\
\end{array}$ & Teste Post-Hoc \\
\hline \multirow{2}{*}{ Clima Exp.Ideias } & & & & $\mathrm{P}_{90}>\mathrm{P}_{25}, \mathrm{p}=0,000$ \\
\hline & 8,615 & $0,000 *$ & 0,195 & $\mathrm{P}_{90}>\mathrm{P}_{50}, \mathrm{p}=0,007$ \\
\hline Interesse Aprend, & 3,526 & $0,017 * *$ & 0,090 & $\mathrm{P}_{90}>\mathrm{P}_{50} ; \mathrm{p}=0,021$ \\
\hline \multirow{4}{*}{ Incentivo Novas Ideias } & & & & $\mathrm{P}_{90}>\mathrm{P}_{25}, \mathrm{p}=0,000$ \\
\hline & & & & $\mathrm{P}_{90}>\mathrm{P}_{50}, \mathrm{p}=0,001$ \\
\hline & 10653 & O OOQ* & 0930 & $\mathrm{P}_{90}>\mathrm{P}_{75}, \mathrm{p}=0,026$ \\
\hline & 10,653 & $0,000^{n}$ & 0,230 & $\mathrm{P}_{75}>\mathrm{P}_{25}, \mathrm{p}=0,033$ \\
\hline $\begin{array}{l}\text { Avaliação e Met. de } \\
\text { Ensino }\end{array}$ & 2,222 & 0,090 & 0,059 & \\
\hline
\end{tabular}

Nota: $* * \mathrm{p}<0,05 ; * \mathrm{p}<0,01$.

Fonte: Elaborado pelos autores.

A partir destas variações nas médias (cf. quadro 2), apresentam-se os resultados da análise de variância multivariada (F-Manova-one way). Assim, a partir dos dados presentes no 
quadro 3, pode-se verificar que os resultados apontam para diferenças estatisticamente significativas em todas as variáveis, exceto na dimensão das práticas criativas Avaliação e Metodologias de Ensino $\left(F=2,222 ; p=0,090, \eta^{2}=0,059\right)$. É de referir ainda que a variação da variável dependente Incentivo a Novas Ideias pode ser explicada em $23 \%$ pela variação do percentil (pessoa mais ou menos criativa), assim como 19,5\% da variação da variável dependente Clima para a Expressão de Ideias. Importa referir também que parte do tamanho do efeito (de ser mais e menos criativo) nas variáveis dependentes práticas criativas (Incentivo a Novas Ideias, Clima para a Expressão de Ideias ou Interesse pela Aprendizagem do Aluno), pode ser atribuída a algumas especificidades desta amostra, nomeadamente ao facto de ser uma amostra de conveniência e ao seu tamanho reduzido.

Para analisar os contrastes múltiplos, optou-se pelo teste de Tukey (1949). Relacionando os resultados da Quadro 3 com a representação gráfica (figura 1), pode-se dizer que relativamente à variável Incentivo a Novas Ideias, os docentes que se situaram percentil 90 contrastam com os docentes que se situam no percentil 25, com os do percentil 50 e com os do percentil 75 (Percentil ${ }_{90}>$ Percentil $_{25}$, Percentil ${ }_{50}$, Percentil $1_{75}$ ), bem como os docentes que se situaram no percentil 75 contrastam face aos do percentil 25 (Percentil $l_{75}>$ Percentil $\left._{25}\right)$. Relativamente à variável Clima de Expressão de Ideias, estes contrastes são verificados entre os docentes do percentil 90 face aos do 25 e do percentil 50 (Percentil ${ }_{90}>$ Percentil $_{25}$, Percentil $\left.{ }_{50}\right)$. Por último, na variável Interesse pela Aprendizagem do Aluno, o contraste acontece apenas entre os docentes do percentil $90 \mathrm{e}$ os que se situam no percentil 50 (Percentil $l_{90}>$ Percentil $\left._{50}\right)$.
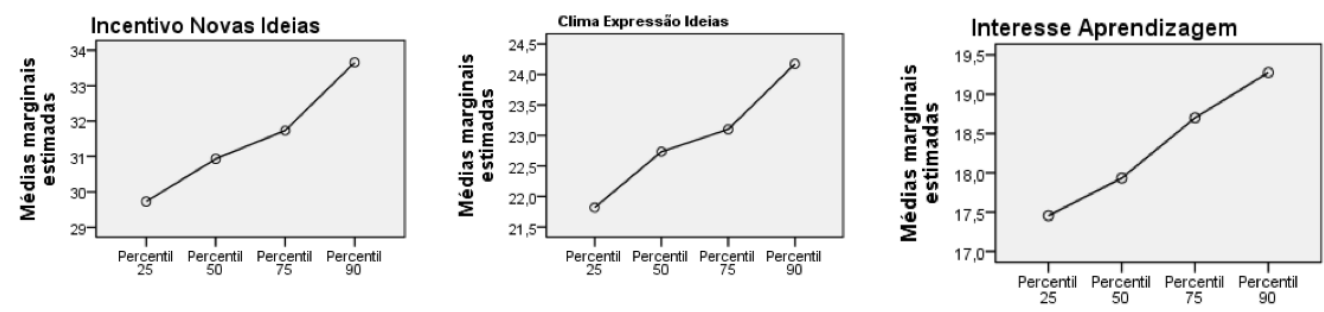

Figura 1. Representação gráfica dos contrastes de acordo com o quartil a que pertence o docente (personalidade criativa)

Fonte: Elaborado pelos autores.

\section{Discussão e conclusões}

Em todos os indivíduos estão disponíveis competências criativas em potencial, podendo estas serem intencionalmente desenvolvidas, nomeadamente no contexto educativo (Alencar, 2015; Ahmadi e Besançon, 2017; Cremin, 2009; Runco 2014). Nesta preocupação com o desenvolvimento criativo dos alunos, o professor surge como essencial enquanto modelo de atitudes e de comportamentos (European Commission, 2015; Glaveanu, 2018). Com base nestes pressupostos, há que refletir quer as práticas que os docentes fomentam na sala de aula em prole da criatividade (Cropley, 2015), quer características pessoais dos mesmos que tornem tais práticas possíveis (Grohaman e Snyder, 2017).

A partir daqui, podem emergir algumas interrogações, nomeadamente sobre a condução de práticas criativas dos docentes no contexto escolar quando se avaliam como mais e menos criativos. Particularmente, neste estudo exploratório interessou analisar se (i) existe uma associação entre as características criativas e práticas criativas docentes e (ii) 
se tais práticas dos docentes variavam em função das suas características mais e menos criativas.

Relativamente ao primeiro objetivo foi possível verificar uma resposta maioritariamente afirmativa: as práticas que os professores exercem em sala de aula, exceto nos aspetos da Avaliação e Metodologias de Ensino, parecem não ser alheias às características de personalidade associadas à criatividade nos docentes. Comentar-se-á a esta associação, e a exceção, quando discutidos os resultados sobre os grupos contrastantes de docentes nas características criativas pessoais, ou seja, tomando também o segundo objetivo que explora e especifica o primeiro.

Quando se toma os vários percentis em personalidade criativa, ainda olhando apenas as médias, observa-se que quanto mais pontuam como criativos, também mais pontuam nas suas práticas criativas no que respeita ao Incentivo a Novas Ideias, ao Clima de Expressão de Ideias e ao Interesse pela Aprendizagem do Alunos. Quando se toma os grupos contrastantes, mantêm-se estas três dimensões das práticas criativas como as que significativamente emergem como distintas entre os docentes mais criativos (percentil 90) e os colegas menos criativos, particularmente no caso do Incentivo a Novas Ideias" (face a todos os restantes percentis) e menos no Interesse pela Aprendizagem do Aluno (apenas face a um dos percentis).

Se há ainda lacunas na investigação empírica sobre práticas criativas em sala de aula e sobre personalidade criativa (Slate et al., 2011; Susanto et al., 2018), torna-se difícil ou mesmo impossível discutir com fundamentação em literatura porque características de personalidade específicas são relevantes para práticas criativas também elas específicas, como no caso de cada uma das três dimensões do Inventário usado no estudo. Os resultados obtidos vêm, sim, reforçar a relação global e positiva entre personalidade criativa do docente e como ele exerce criativamente no ensino aprendizagem, havendo influência da primeira variável na segunda. Esta perspetiva parece encontrar eco em autores como Piirto (2011), Gladilina (2016) ou Jauk e colegas (Jauk, 2019, Jauk, Benedek e Neubauer, 2014). Aliás, esta ideia de que um indivíduo criativo é fator chave para aumentar ou obstrui possibilidades de um clima que, por sua vez, desenvolve ou não a expressão da criatividade, era já afirmada por MacKinnon (1978). Alguém criativo ou, neste caso, um docente criativo no sentido que Craft (2005) afirmava, não se esgota certamente na personalidade criativa que possui e necessita rentabilizar recursos externos para promover criatividade dos seus alunos. Porém, um conjunto de características como motivação intrínseca, abertura à experiência, autonomia, persistência ou tolerância à ambiguidade parecem ter impacto na qualidade de um ensino que o docente operacionaliza no seu quotidiano profissional de forma a promover competências criativas nos seus alunos.

Reportando às dimensões de práticas criativas avaliadas neste estudo, para incentivar os alunos a ter novas ideias e para criar um clima que o leve a expressá-las, será necessárias características como autoconfiança, elevada motivação, apetência pelo risco ou abertura à experiência. Por exemplo, esta última característica tem sido considerada como uma das mais distintivas numa personalidade criativa para fomentar um clima criativo (Jauk et al., 2014). Já para ter e demonstrar interesse pela aprendizagem dos alunos, não será tão óbvio o requisito de tal característica, mas certamente são importantes outras de uma personalidade criativa como a motivação intrínseca, a persistência ou a atração por desafios (Morais e Fleith, 2017). Como referem Navickiene e colegas (2019), ser um 
professor criativo será relevante para aspetos como o domínio e o entusiasmo face ao que leciona, a qualidade de interação com os seus alunos, a abordagem do que é criatividade, a condução de tarefas que tenham a ver com os interesses do aluno, ou seja, características subjacentes às três dimensões aqui analisadas.

Pode-se ainda olhar as dimensões que neste estudo emergiram como extremas, ou seja, o Incentivo a Novas Ideias como a que parece requerer mais a criatividade no docente e a Avaliação e Metodologias de Ensino que surge como alheia a essa variável. No primeiro caso, não parece surpreendente que professores mais criativos tentem desenvolver em sala de aula aspetos dos mais associados a criatividade: a novidade e a divergência de ideias. $\mathrm{O}$ próprio conceito de criatividade exige a aliança da eficácia das soluções/ideias à originalidade (Runco, 2014) e a divergência foi sempre inerente à conceção de processo criativo (Guilford, 1987; Isaksen, Dorval e Treffinger, 2011). No outro caso, apesar de se saber a relevância do desenvolvimento da criatividade no contexto escolar, ainda é frequente a reclamação de que este contexto continua a obstrui-la com metodologias e tarefas que pautam pela repetição, estandardização ou memorização, muitas vezes aliadas a aspetos organizacionais e mais dificilmente ultrapassáveis pelos docentes (Cropley, 2015; Fleith e Morais, 2017; MacLaren, 2012) pode ser este o caso específico da avaliação e das metodologias de ensino. Resta a curiosidade, apesar de esta não se apoiar em dados estatisticamente significativos (valores das médias), de porque os docentes menos criativos (percentil 25) avaliam mais positivamente as suas práticas em Avaliação e metodologias de Ensino face aos colegas, nomeadamente face aos mais criativos (percentil 90). Estes últimos docentes serão mais exigentes na avaliação de criatividade que fazem das suas práticas mais institucionais? Os menos criativos entenderão esta dimensão como a mais fácil de contornar desde que haja a tentativa da não repetição de rotinas institucionais e nada mais? Seguramente, são especulações que podem e devem estimular mais investigação.

Este estudo teve um caráter ainda exploratório e podem ser referidas algumas limitações. Em primeiro lugar, foi trabalhada uma amostra não probabilística a partir de um grupo de trabalho de docentes que já integrava uma investigação-ação e houve grande disparidade entre o número de homens e mulheres. É de recordar ainda que esta pesquisa recorreu à avaliação de ambas as variáveis em causa a partir de autorrelatos/autoavaliações dos professores, com a subjetividade e o que tal tipo de medidas implica (McGrath et al., 2010; Tracey, 2016). Relativamente aos instrumentos de recolha de informação, pode-se referir que não se conhecem estudos de validação específica com docentes tomando a Escala de Personalidade Criativa e que não há estudos comparativos entre a aplicação do Inventário de Práticas para Criatividade entre docentes universitários (versão original) e do Ensinos Básico. Por outro lado, o tipo de pesquisa é desafiado por questões metodológicas associadas aos desenhos transversais e à variância do método comum. Em pesquisas futuras, haverá então a necessidade de aumentar o tamanho da amostra, a tipologia probabilística, assim como considerar, além das perceções, a realização criativa do docente a avaliar e as suas práticas no contex to educativo, nomeadamente pela observação destas últimas. Os resultados não podem, consequentemente, ser já generalizáveis.

Este estudo de cariz exploratório sugere, porém, uma perspetiva a ser explorada: docentes que se consideram pouco e muito criativos surgem avaliando-se diferentemente nas práticas que favorecem a criatividade dos seus alunos e, tendencialmente, os mais criativos parecem mais inovadores em algumas dimensões destas práticas. Assim, se nem todas as facetas das práticas pedagógicas para a criatividade parecem condicionáveis pela 
personalidade criativa do docente (numa interação entre o docente e características mais estáticas, organizacionais, por exemplo, como a avaliação ou a estrutura e exigências de lecionação), a personalidade criativa aparece como um recurso para o exercício de outras práticas com esse objetivo de fomentar a criatividade. Estas reflexões deverão ser mais estudadas em futuras pesquisas e serem consideradas nos espaços de formação de professores, grupo este privilegiado para a concretização do desenvolvimento de cidadãos futuros mais criativos e inovadores (Ahmadi e Besançon, 2017; European Commission, 2015).

\section{Referências}

Ahmadi, N. e Besançon, M. (2017). Creativity as a stepping stone towards developing other competencies in classrooms. Education Research International, 34, 56-77. https://doi.org/10.1155/2017/1357456

Alencar, E. M. (2015). Promoção da criatividade em distintos contextos: Entraves e desafios. En M. F. Morais, L. C. Miranda e S. M. Wechsler (Orgs.), Criatividade: Aplicações práticas em contextos internacionais (pp. 59-136). Vetor.

Alencar, E. M. L. S. e Fleith, D. S. (2010). Inventário de práticas docentes para a criatividade na educação superior. En E. M. L. S. Alencar, M. F. Bruno-Faria e D. S. Fleith (Orgs.), Medidas de criatividade. Teoria e prática (pp. 71-89). ARTMED.

Alencar, E. M. L. S. e Sobrinho, A. B. F. (2017). A gestão da criatividade. PRISMAS.

Amabile, T. M. (1983). The social psychology of creativity. Prentice-Hall. https://doi.org/10.1007/978-1-4612-5533-8

Amabile, T. M. (1996). Creativity in context: Update the social psychology of creativity. Westview Press.

Almeida, L. S. e Freire, T. (2017). Metodologia da investigação em psicologia da educação. Psiquilíbrios.

Andreas, S. F. K., Zech, S., Coyle, T. R. e Rindermann, H. (2016). Unconventionality and originality: Does self-assessed unconventionality increase original achievement? Creativity Research Journal, 28(2), 198-206. https://doi.org/10.1080/10400419.2016.1162556

Azevedo, I., Morais, M. F. e Martins, F. (2017). Educação para a criatividade em adolescentes: Uma experiência com o future problem solving program internacional. REICE. Revista Iberoamericana sobre Calidad Eficacia y Cambio en Educacion 15(2), 75-87. https://doi.org/10.15366/reice2017.15.2.004

Baer, J. (2017). Content matters: Why nurturing creativity is so different in different domains. En R. A. Beghetto e B. Sriraman (Eds.), Creative contradictions in education: Cross disciplinary paradoxes and perspectives (pp. 129-140). Springer. https://doi.org/10.1007/978-3-319-21924-0_8

Barbot, B., Besançon, M. e Lubart, T. (2015). Creative potential in educational settings: Its nature, measure, and nurture. International Journal of Primary and Early Years 43(4), 371-381. https://doi. org/10.1080/03004279.2015.1020643

Barron, F. (1969). Creative person and the creative process. Holt, Rinehart \& Winston.

Batey, M. e Hughes, D. J. (2017). Individual difference correlates of self-perceptions of creativity. En M. Karwowski e J. Kaufman (Eds.). The creative self(pp. 184-217). Academic Press. https://doi.org/10.1016/B978-0-12-809790-8.00011-X

Beghetto, R. A. e Kaufman, J. C. (2014). Classrooms contexts for creativity. High Ability Studies, 25(1), 53-69. https://doi.org/10.1080/13598139.2014.905247 
Catarino, P, Vasco, P., Lopes, J., Silva, H. e Morais, E. (2019). Cooperative learning on promoting creative thinking and mathematical creativity in higher education. REICE. Revista Iberoamericana sobre Calidad, Eficacia y Cambio en Educación, 17(3), 5-22. https://doi.org/10.15366/reice2019.17.3.001

Ceci, M. W. e Kumar, V. K. (2016). A correlational study of creativity, happiness, motivation, and stress from creative pursuits. Journal of Happiness Studies,15(6) 609-626. https://doi. org/10.1007/s10902-015-9615-y.

Chávez-Eakle, R. A., Eakle, A. J. e Cruz-Fuentes, C. (2012). The multiple relations between creativity and personality. Creativity Research Journal, 24(1), 76-82. https://doi. org/10.1080/10400419.2012.649233

Costa, S., Páez, D., Sánchez, F., Garaigordobil, M. e Gondim, S. (2015). Personal factors of creativity: A second order meta-analysis. Journal of Work and Organizational Psychology, 31(3), 165-173. https://doi. org/10.1016/j.rpto.2015.06.002

Craft, A. (2005). Creativity in schools: Tensions and dilemmas. Routledge. https://doi.org/10.4324/9780203357965

Craft, A. e Jeffrey, B. (2010). Creativity and performativity in teaching and learning. British Educational Research Journal, 34(5), 577-584. https://doi.org/10.1080/01411920802223842

Cremin, T. (2009). Creative teachers and creative teaching. En A. Wilson(Ed.), Creativity in primary education (pp. 36-46). Learning Matters.

Cropley, A. (2015). Creativity in education and learning. A guide for teachers and educators. Routledge. https://doi.org/10.4324/9780203826270

Cropley, A. e Cropley, D. (2009). Fostering creativity: A diagnostic approach for higher education and organizations. Hampton Press.

Csíkszentmihályi, M. (2014). Creativity: Flow and the psychology of discovery and invention. Harper Perennial.

Davidovitch, N. e Milgram, R. M. (2006). Creative thinking as a predictor of teacher effectiveness in higher education. Creativity Research Journal, 18(3) 385-390.

https://doi.org/10.1207/s15326934crj1803_12

European Commission. (2015). Unleashing young people's creativity and innovation. European good practice projects. Publications Office of the European Union. https://doi.org/ 10.2766/8245

Feist, G. J. (2017). The creative personality. Current understandings and debates. En J. A. Plucker (Ed.), Creativity and innovation: Theory, research and practice (pp.181-199). Prufrock Press. https://doi.org/10.1007/978-1-4614-6616-1_185-2

Fleith, D. S. e Alencar, E. M. (2005). Percepção de alunos do ensino fundamental quanto ao clima de sala de aula para criatividade. Psicologia em Estudo, 11(3), 513-521. https://doi.org/10.1590/S1413-73722006000300007

Fleith, D. S. e Morais, M. F. (2017). Desenvolvimento e promoção da criatividade. En L. S. Almeida (Ed.), Criatividade e pensamento crítico: Conceito, avaliação e desenvolvimento (pp. 45-73). CERPSI.

Garcês, S., Pocinho, M., Jesus, S., Viseu, J., Imaginário, S. e Wechsler, S. M. (2015). Estudo de validação da escala de personalidade criativa. Revista Iberoamericana de Diagnóstico e Evaluación, 40(2), 17-24.

Gladilina, I. P. (2016). Creativity in the structure of professionalism of a higher school teacher. International Journal of Environmental and Science Education, 11(8), 1691-1699. 
Glaveanu, V. P. (2018). Educating which creativity? Thinking Skills and Creativity, 27(1), 25-32. https://doi.org/ 10.1016/j.tsc.2017.11.006

Gough, H. G. (1979). A creative personality scale for the adjective check list. Journal of Personality and Social Psychology, 37(8), 1398-1405. https://doi.org/10.1037/0022-3514.37.8.1398

Grohman, M. G. e Snyder, H. T. (2017). Why do we create? The roles of motivation, mindset, and passion in human creativity. En J. A. Plucker (Ed.), Creativity and innovation: Theory, research, and practice (pp. 165-180). Prufrock Press.

Guilford, J. P. (1967). The nature of human intelligence. MacGraw-Hill.

Higuchi, T., Yuizono, T., Miyata, K., Sakurai, K. e Kawaji, T. (2016). Creativity effects of ideamarathon system: Torrance tests of creative thinking. En A. M. J. Skulimowski e J. Kacprzyk (Eds.), Knowledge, information and creativity support systems: Recent trends, advances and solutions (pp. 185-200). Springer. https://doi.org/10.1007/978-3-319-19090-7_15

Isaksen, S., Dorval, K. e Treffinger, D. (2011). Creative approaches to problem solving: A framework for change. Kendall Hunt.

Kampylis, P. e Berki, E. (2014). Nurturing creative thinking. UNESCO.

Kaufman, J. C. (2016). Creativity 101. Singer Publishing Company. https://doi.org/10.1891/9780826129536

Kaufman, J. e Beghetto, R. (2008). Exploring mini c: Creativity across cultures. En R. DeHaan, K. M. Narayan (Ed.), Education for innovation in India, China and America (pp. 165-180). Brill Sense. https://doi.org/10.1163/9789087902858_009

Kaufman, B. e Gregoire, C. (2015). Wired to create: Unraveling the mysteries of the creative mind. Perigee.

Lambert, P. A. (2017). Understanding creativity. En J. B. Cummings e M. L. Blatherwick (Eds.), Creative dimensions in teaching and learning in the 21st century. Advances in creativity and Giftedness (pp.1-2 1). Sense Publishers. https://doi.org/10.1007/978-94-6351-047-9_1

Lubart, T. e Zenasni, F. (2010). A new look at creative giftedness. Gifted and Talented International, 25, 53-57. https://doi.org/10.1080/15332276.2010.11673549

Jauk, E., Benedek, M. e Neubauer, A. C. (2014). The road to creative achievement: A latent variable model of ability and personality predictors. European Journal of Personality, 28(1), 95-105. https://doi.org/10.1002/per.1941

Jauk, E., Eberhardt, L., Koschmieder, C. Diedrich, J., Pretsch, J., Benedek, M. e Neubauer, A. C. (2019). A new measure for the assessment of appreciation for creative personality. Creativity Research Journal, 31(2), 149-163. https://doi.org/10.1080/10400419.2019.1606622

MacKinnon, D. W. (1978). In search of human effectiveness: Identifying and developing creativity. Creative Education Association.

MacLaren, I. (2012). The contradictions of policy and practice: Creativity in higher education. London Review of Education, 10 (2), 159-172. https://doi.org/10.1080/14748460.2012.691281

McGrath, R. E., Mitchell, M., Kim, B. H. e Hough, L. (2010). Evidence for response bias as a source of error variance in applied assessment. Psychological Bulletin, 136(3), 450-70. https://doi.org/ 10.1037/a0019216

Miller, A. L. e Dumford, A. D. (2016). Creative cognitive processes in higher education. The Journal of Creative Behaviour, 50(4) 282-293. https://doi.org/10.1002/jocb.77 
Ministério da Educação. (2017). Perfil dos alunos à saída da escolaridade obrigatória. Ministério da Educação.

Miranda, L. e Morais, M. F. (2019). Criatividade e motivação: Um estudo exploratório em docentes. Revista de Estudios e Investigación en Psicología y Educación, 6(2), 114-125. https://doi.org/10.17979/reipe.2019.6.2.5277

Moeller, J., Keiner, M. e Grassinger, R. (2015). Two side of the same coin: Do the dual types of passion describe districts' subgroups of individuals? Journal for Person-Oriented Research, 1(3), 131-150. https://doi.org/10.17505/jpor.2015.15

Monteiro, I., Morais, M. F. e Martins, F. (2020). Desenvolvimento de competências criativas: Um estudo com alunos angolanos do ensino primário. Revista EDUCAmazônia-Educação, Sociedade e Meio Ambiente, 25(2), 250-275.

Morais, M. F. (2013). Creativity: Challenges to a key-concept for the twenty-first century. En A. Antonietti, B. Colombo e D. Memmert (Eds.), Psychology of creativity: Advances in theory, research and application (pp. 3-19). NOVA Publisher.

Morais, M. F. e Almeida, L. (2019). I would be more creative if...: Are there perceived barriers to college students' creative expression according to gender? Estudos de Psicologia, 36, 11-23. https://doi.org/10.1590/1982-0275201936e180011

Morais, M. F. e Fleith, D. (2017). Conceito e avaliação de criatividade. En L. Almeida (Coord.), Criatividade e pensamento crítico: Conceito, avaliação e desenvolvimento (pp.19-44). CERPSI.

Morais, M. F., Almeida, L. S., Azevedo, I., Alencar, E. S. e Fleith, D. S. (2014). Validação portuguesa do inventário de práticas docentes para criatividade na educação superior. Avaliação Psicológica, 13(2), 167-175.

Morais, M. F., Viana, F. L., Fleith, D. e Dias, C. (2019) Portuguese adaptation of the classroom climate for creativity scale.Temas em Psicologia/Trends in Psychology, 27(4), 837-849. https://doi.org/10.9788/tp2019.4-02 .

Muñoz-Pascual, L. e Galende, J. (2017). The impact of knowledge and motivation management on creativity: Employees of innovative Spanish companies. Employee Relations, 39(5), 732-752. https://doi.org/10.1108/ER-05-2016-0096

Navickiene, V., Sedereviciute-Paciauskiene, Z., Valantinaite, I. e Zilinskaite-Vitiene, V. (2019). The relaship betwen communication and education through the creative personality of the teacher. Creativity Studies, 12(1), 49-60. https://doi.org/103846/cs.2019.6472

Omdal, S. e Graefe, A. K. (2017). Investing in creativity in students: The long and short (term) of it. En J. A. Plucker (Ed.), Creativity and innovation. Theory, research, and practice (pp. 205-221). Prufrock Press.

Patston, T. J., Cropley, D., Marrone, R. L. e Kaufman, J. C. (2017). Teacher self-concepts of creativity: Meeting the challenges of the 21 st century classroom. The International Journal of Creativity \& Problem Solving, 27(2), 23-34.

Piirto, J. (2011) Creativity for 21st century-how embed creativity into the curriculum. Sense Publishers. https://doi.org/10.1007/978-94-6091-463-8

Raso, F. e Arando, D. S. (2019). Percepciones del futuro pedagogo sobre la metodología de enseñanza de la creatividad REICE. Revista Iberoamericana sobre Calidad, Eficacia y Cambio en Educación, 17(1), 73-89. https://doi.org/10.15366/reice2019.17.1.005

Robinson, J. C., Workman, J. e Freeburg, B. W. (2018). Creativity and tolerance of ambiguity in fashion design students. International Journal of Fashion Design Technology and Education, 12(1), 1-9. https://doi.org/10.1080/17543266.2018.1516807 
Runco, M. A. (2014). Creativity: Theories and themes. Academic Press.

Runco, M. e Jager, G. (2012). The standard definition of creativity. Creativity Research Journal, 24(1), 92-96. https://doi.org/10.1080/10400419.2012.650092

Ryan, R. M. e Deci, E. L. (2017). Self-determination theory: Basic psychological needs in motivation, development, and wellness. Guilford Publishing. https://doi.org/10.1521/978.14625/28806

Sierra, M. D., Escobedo, P. S., Cuervo, A. A. V. e Rosal, A. B. (2015). Estratégias e programas para o desenvolvimento da criatividade nas escolas do México. En M. F. Morais, L. C. Miranda e S. M. Wechsler (Eds.), Criatividade. Aplicações práticas em contextos internacionais, (pp. 257268). Vetor.

Sadeghi, A. e Ofoghi, N. (2011). The psychological factors affecting student's creativity inside the class. Procedia Social and Behavioural Sciences, 15, 263-270.

https://doi.org/10.1016/j.sbspro.2011.03.084

Seckel, M. J., Breda, A., Sánchez, A. e Font, V. (2019). Criterios asumidos por profesores cuando argumentan sobre la creatividad matemática. Educação e Pesquisa, 45, e211926.

https://doi.org/10.1590/s1678-4634201945211926

Slate, J., LaPrairie, K. N., Schulte, D. P. e Onwuegbuzie, A. (2011). Views of effective college faculty: A mixed analysis. Assessment and Evaluation in Higher Education, 36(3), 331-346. https://doi.org/10.1080/02602930903428684

Soulé, H. e Warrick, T. (2015). Defining 21 st century readiness for all students: What we know and how to get there. Psychology of Aesthetics, Creativity, and the Arts, 9(2), 178-186.

https://doi.org/10.1037/aca0000017

Starko, A. J. (2014). Creativity in the classroom-schools of curious delight. Routledge. https://doi.org/10.4324/9780203115176

Susanto, E., Novitasari, Y., Yusuf, S. e Ilfianfdra, I. (2018). Development of creative personality inventory: Hypothetical concept. International Journal of Innovation, Creativity and Change, 4(2), 176-192. https://doi.org/10.31227/osf.io/fnp87

Tracey, T. J. G. (2016). A note on socially desirable responding. Journal of Counseling Psychology, 63(2), 224-32. https://doi.org/10.1037/cou0000135.

Tukey, J.W. (1949). Comparing individual means in the analysis of variance. Biometrics, 5, 99-114. https://doi.org/10.2307/3001913

Williams, R., Runco, M. e Berlow, E. (2016). Mapping the theme, impact, and cohesion of creativity research over the last 25 years. Creativity Research Journal, 28(4), 385-394. https://doi.org/10.1080/10400419.2016.1230358

\section{Agradecimientos}

Este trabalho é financiado pelo CIEd-Centro de Investigação em Educação, Instituto de Educação, Universidade do Minho, projetos UIDB/01661/2020 e UIDP/01661/2020, através de fundos nacionais da FCT/MCTES-PT. 


\section{Breve CV dos autores}

\section{Maria de Fátima Morais}

Licenciada em Psicologia (Universidade do Porto, Portugal) e doutorada em Psicologia da Educação (Universidade do Minho- Braga, Portugal). É Professora Auxiliar no Deparatmento de Psicologia da Educação e de Educação Especial. É membro investigadora integrada no CIEd (Centro de Investigação e Educação da Universidade do Minho). Frequentemente realiza cursos de graduação e de pos graduação acerca de criatividade, resolução de problemas e inovação. É membro da REINEVA (Rede Internacional de Pesquisa, Avaliação e Intervenção em Altas capacidades) e da ANEIS (Associação Nacional de Estudos e Intervenção na Sobredotação). ORCID ID: https://orcid.org/0000-0001-8180-6687. Email: famorais@ie.uminho.pt

\section{Lúcia C. Miranda}

Psicóloga e mestre pela Universidade de Coimbra, doutorada e pós-doutorada pela Universidade do Minho na área de especialização Psicologia da Educação. Psicóloga Especialista pela Ordem dos Psicólogos Portugueses em Psicologia da Educação e em Psicologia Social e das Organizações. Presentemente é professora auxiliar convidada na Universidade da Madeira, Departamento de Psicologia. Colabora como investigadora com o Centro de Investigação em Educação (CIEd) na Universidade do Minho. ORCID ID https://orcid.org/0000-0002-0652-0811. Email: lrcmiranda@gmail.com 